\title{
Viscoelastic Properties of Edematous Skin
}

\author{
Yawara YOSHITOSHI, $M_{;}$D. and \\ Masahito NAGASAKA, M.D.
}

\begin{abstract}
A continuously recording apparatus is devised to measure the deformation of skin surface on tibial margin against test weight. The deformation observed by this apparatus is best approximated by 4 element viscoelastic model in rheology. One of the moduli, $\eta_{1}$, is thought to represent capillary filtrability.

In edematous patients there are seen profound alterations in these moduli, and an increased capillary filtrability and easy susceptibility to deformation are expressed quantitatively. After dissipation of edema these alterations usually return to normal range.
\end{abstract}

$\mathbf{I}^{\mathrm{T}}$ $T$ is difficult to measure the degree of peripheral edema in quantity and it is usually checked by the appearance of impression caused by finger pressure. In 1926, Schade invented elastometry to measure the grade of edema and succeeded to express numerically his elasticity-loss in accordance with the severity of the edema.1) The measurement of elasticity-loss is, in brief, to calculate the ratio of sink and upheaval of skin surface after each 2 minutes of application and removal of test weight. Watanabe, in 1953, re-examined the elastometry and defined another sign for classification of edema. ${ }^{2}$ He measured increased fluidity beside elasticity-loss of the skin and termed the method as sclerometry. We found that these measurements could be reinterpreted in terms of rheology, and proposed more physiologically usable moduli for quantification of degree of the cdema. ${ }^{3)}$.

\section{METHODS}

\section{Apparatus :}

The displacement of moving rod is electrically recorded on a pen-oscillograph. Transducer is a specially designed differential-transformer,* and the moving rod is supported by pick-up (of record player) type arm.** The weight

From Prof. Yoshitoshi's Clinic of Internal Medicine, Faculty of Mcdicinz, University of Tokyo, Tokyo, Japan.

* Transformer is supplied from Shinko Electric Work.

** The apparatus is manufactured by Sugiura Laboratory Work. 
of rod can be balanced by appropriate counterweight at will. The rod has a diameter of $3 \mathrm{~mm}$. and shaped round, so its cross-sectional area is about $7 \mathrm{~mm} .^{2}$ We determined the effective weight of rod to be $3 \mathrm{Gm}$. and this is thought to be satisfactory for avoiding unnecessary oscillation at the time of removing the test weight. The pressure just beneath the rod is calculated to be about $30 \mathrm{~mm} . \mathrm{Hg}$. Before the test, the rod is kept by trigger mechanism, and so the pressures of sink and upheaval of the skin are not strictly reciprocal. The frequency response of the arm is proved to be sufficient for the measurement. Apparatus is shown in Fig. 1.

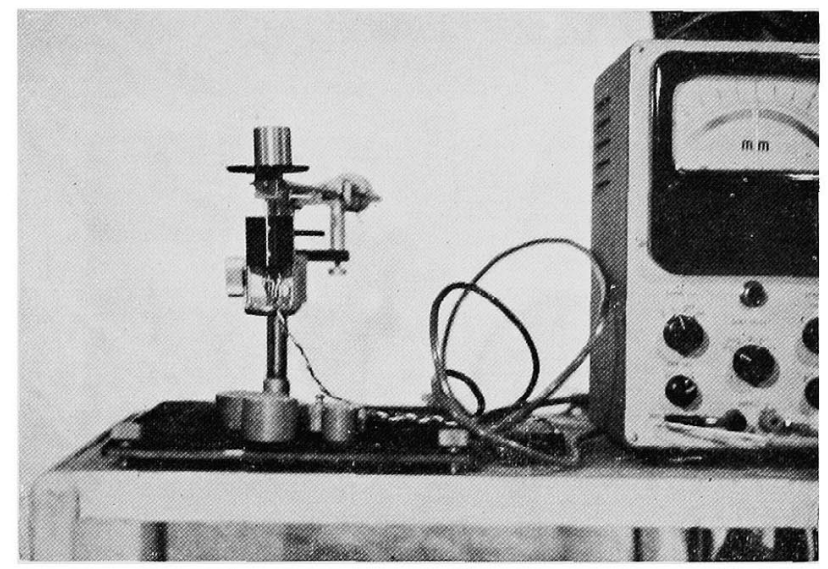

Fig. 1. Appearance of the apparatus.

\section{Exprimeralal procudure:}

The apparatus is placed on the right foot of patients in recumbent position and the rod is brought in close contact with the skin surface on tibial margin. The foot is loosely kept in fixed position by cushions. Test weights are 20, 50, 100, 200 and $300 \mathrm{Gm}$. and a different part of the skin surface is employed for each weight. The recordings are made 2 minutes for sink and then 2 minutes for upheaval. In some cases, recordings are repeated for the same weight, and comparable records are obtained although they are not so strictly reproducible because of the inequality of skin thickness and possibly also of its composition. The removal of test weight is performed manually, and does not require any special skill to eliminate artefact. Of course some oscillations are inevitably recorded, but they subside so immediately that they do not produce any grave annoyance for measurement. Two specimens of records are reproduced for illustration (Fig. 2 and 3).

\section{Calculation of: moduli:}

Before moduli are measured and calculated, adequate rheological model must be determined from obtained curves. As is well known, rheology can not make up a unique model from any recorded responses of complex nature. We decided to use 4 element model (Maxwell-Voigt type) which seems to be most suitable for our analysis (Fig. 4 and 5). The reasoning why we choose this model is discussed in later part of this article.

Once model is determined, measurement is easy. Keys to be found at first are flexion point which shows the end of the effect of $\mathrm{E}_{1}$, and asymptotic line 


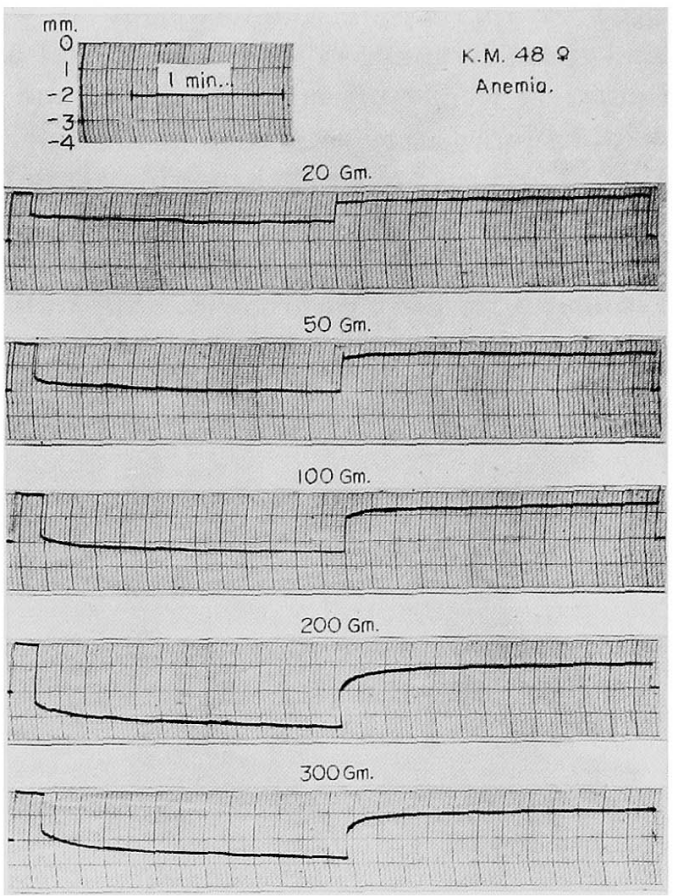

Fig. 2. Rheogram in human foot. Non-edematous patient.

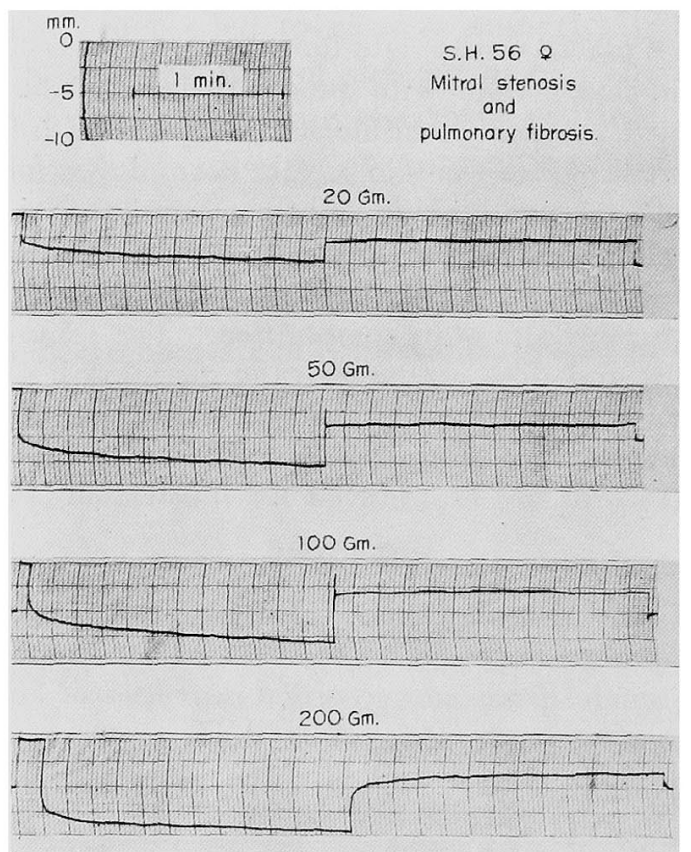

Fig. 3. Rheogram in human foot. Edematous patient. 


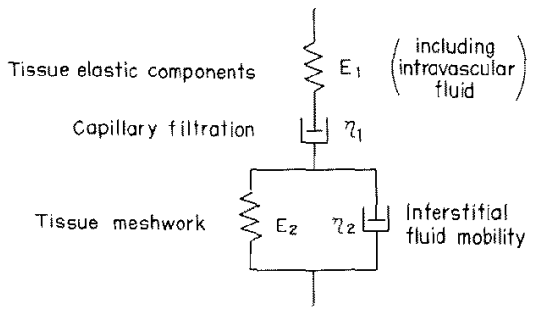

Fig. 4. Tentative correspondence of viscoelastic elements of skin.

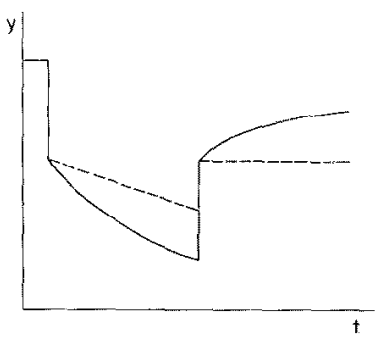

$$
\begin{aligned}
\frac{d^{2} F}{d t^{2}}+ & \left(\frac{E_{1}}{\eta_{1}}+\frac{E_{1}}{\eta_{2}}+\frac{E_{2}}{\eta_{2}}\right) \frac{d F}{d t}+\frac{E_{1} E_{2}}{\eta_{1} \eta_{2}} F \\
= & E_{1} \frac{d^{2} y}{d t^{2}}+\frac{E_{1} E_{2}}{\eta_{2}} \frac{d y}{d t} \\
y= & \left(\frac{1}{E_{1}}+\frac{1}{\eta_{1}} t\right) Y(t) \\
& +\frac{1}{E_{2}}\left\{1-\exp \left\{-\frac{E_{2}}{\eta_{2}} t\right)\right\} Y(t) \\
& Y(t): \text { step function(direction inverted) }
\end{aligned}
$$

Fig. 5. Response determined by the model of Fig. 4.

which shows the effect of summation of $r_{1}$ and $\mathrm{E}_{2}$. $\eta_{2}$ needs to be calculated graphically from time constant of logistic part of curves between asymptotic line and its parallel that passes the flexion point, according to the relation: $\mathrm{T}=\gamma_{2} / \mathrm{E}_{2} . \quad \gamma_{1}$ is slope determined by 2 straight lines passing the flexion point, one parallel to the base line and the other parallel to the asymptotic line. We will mention later that we often found the need for a more generalized Voigt model which seems to have at least 2 viscoelastic time constants. But the subsidence of the quicker component is usually early enough, that we can make this component included in $\mathrm{E}_{i}$. It is, of course, an approximation, nevertheless we get ample information from thus determined 4 moduli.

\section{Results}

\section{Moduli of non-edematous patients.}

In 15 male and 8 female patients of various diseases, 4 moduli were measured according to the calculation as described above. Each modulius was expressed in its reciprocal for convenience of intuitive understanding. The reciprocals sometimes have their own names and symbols, but we left customary usage of nomenclature because of their popularity. The distributions of moduli are demonstrated in Fig. $6 a-d$ and $7 a-d$, separating male and female patients. Instead of mean value, central value was employed for representative value for each modulus. 

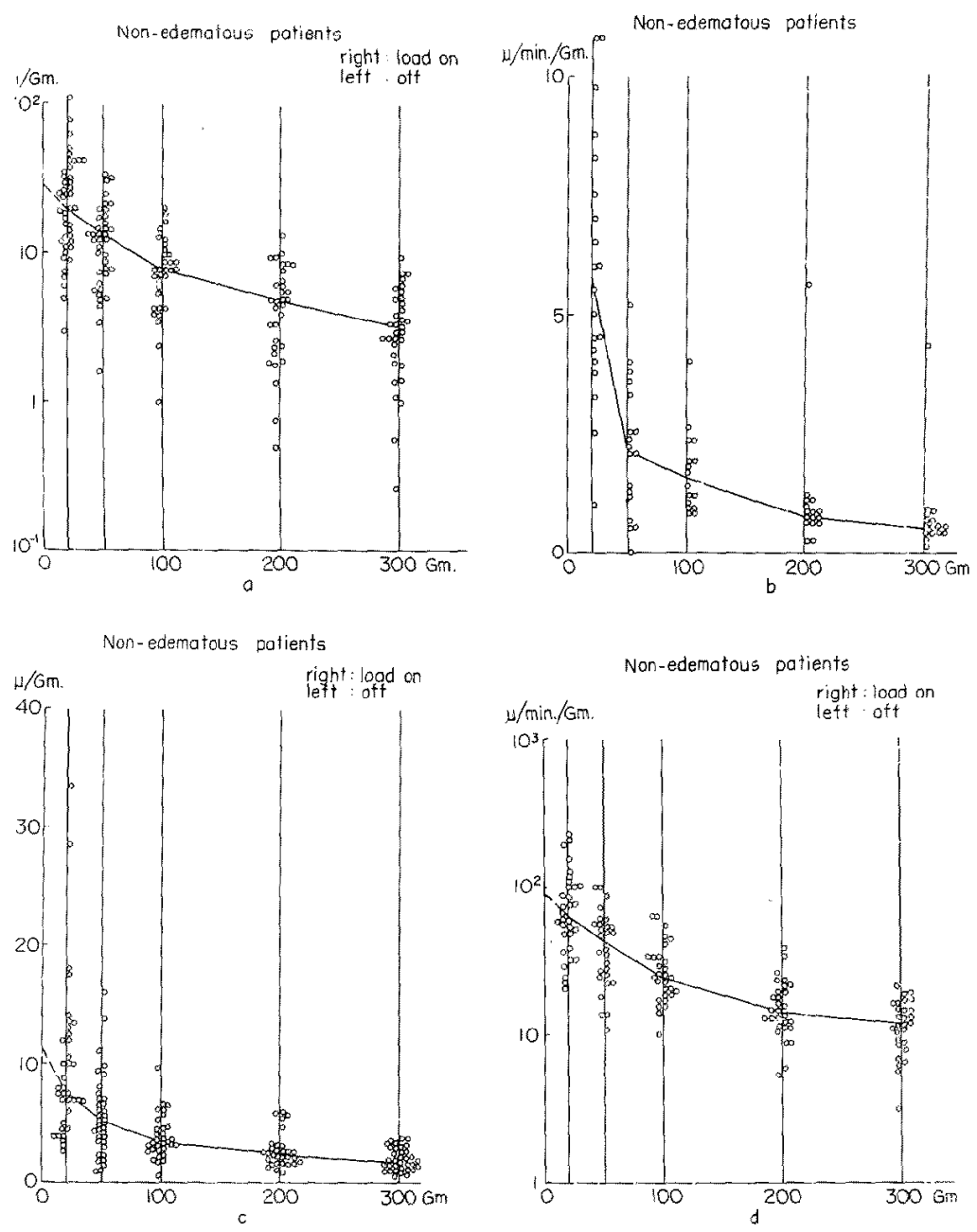

Fig. 6 a. Distribution of $1 / \mathrm{E}_{1}$ in human male.

b. Distribution of $1 / \eta_{1}$ in human male.

c. Distribution of $1 / E_{2}$ in human male.

d. Distribution of $1 / \eta_{2}$ in human male.

Because of wide dispersion of the age and the disease, we felt inadequacy of making mean of each modulus. On and off values of responses to test weight were usually not identical. One of the reasons was already mentioned above.

Most apparent feature of measured moduli was their non-linearity for the test weight. Schade also mentioned this effect and attributed it to the structural deformation as the result of too heavy load. ${ }^{1)}$ For some moduli simple extrapolations for zero load could be made. But we chose rather value for $20 \mathrm{Gm}$. as representative of modulus. Separation of male and 

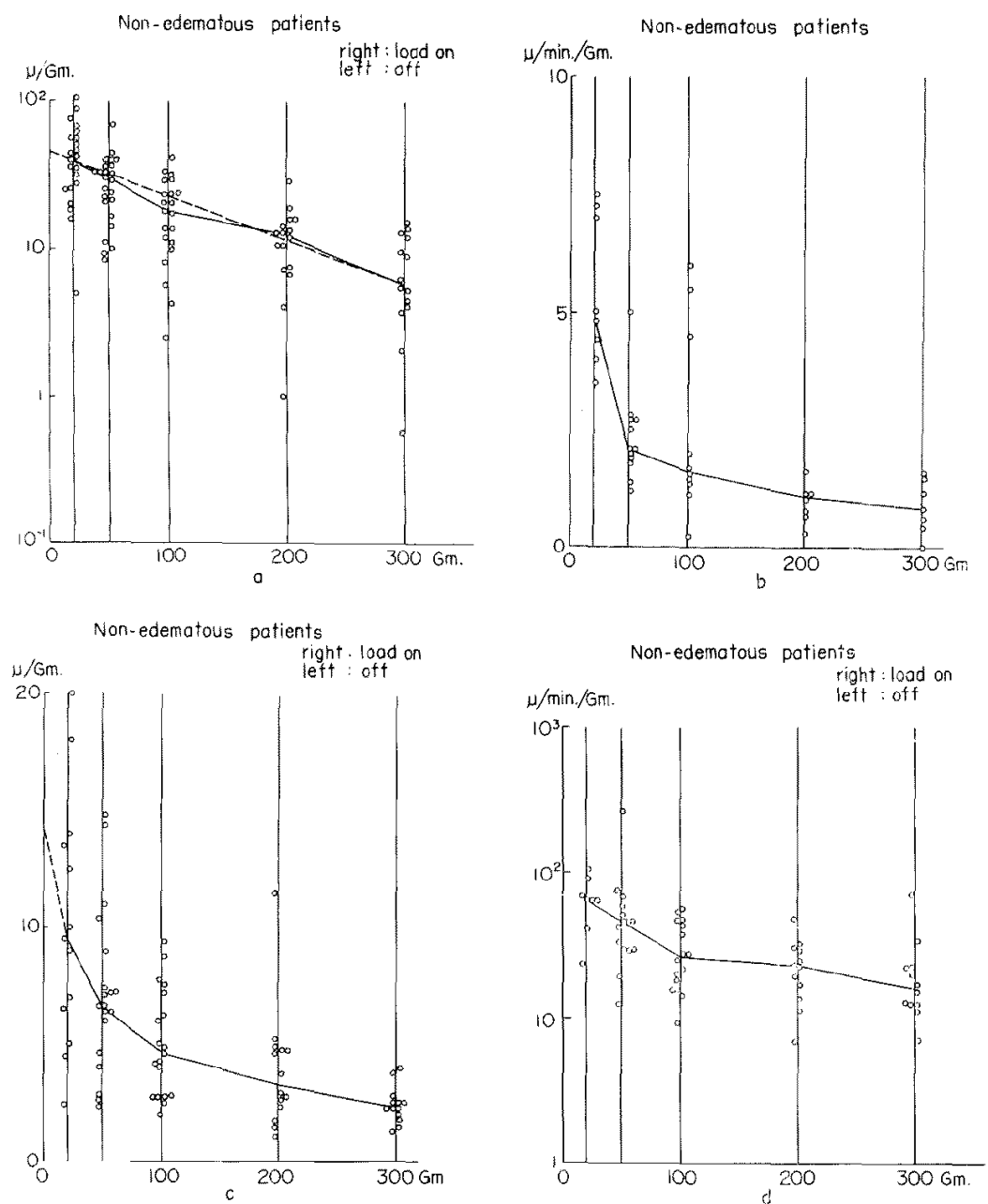

Fig. 7 a. Distribution of $1 / E_{1}$ in human female.

b. Distribution of $1 / \eta_{1}$ in human female.

c. Distribution of $1 / \mathrm{E}_{2}$ in human female.

d. Distribution of $1 / \eta_{2}$ in human female.

female patients was effective in elucidating the difference of moduli between genders (Fig. 8). The differences were most distinct in $\mathrm{E}_{1}$ and $\mathrm{E}_{2}$. This naturally means that the skin of female is more elastic and more susceptible for deformation by external force than that of male.

Moduli of edematous patients.

In 3 slightly edematous and 2 heavily edematous female patients, 4 moduli were also calculated and demonstrated in Fig. 9a and b. Nonedematous central values were plotted in the graph for the comparison. It is easily conceivable that the fluidity of edematous tissue is greater than 


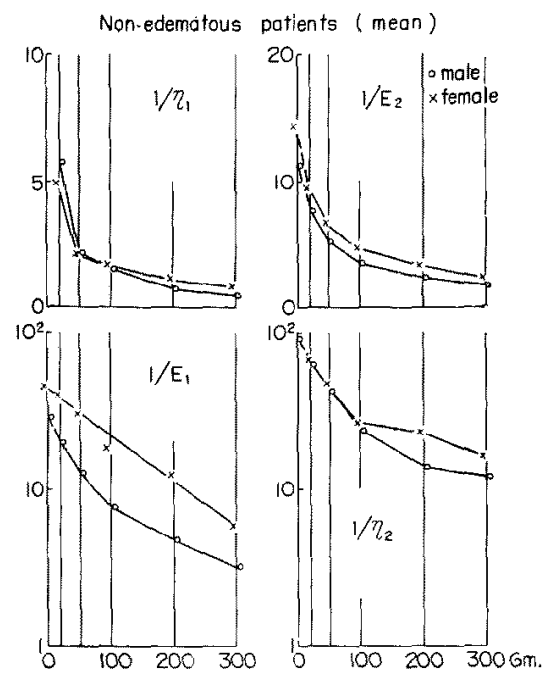

Fig. 8. Comparison of viscoelastic moduli of male and female.
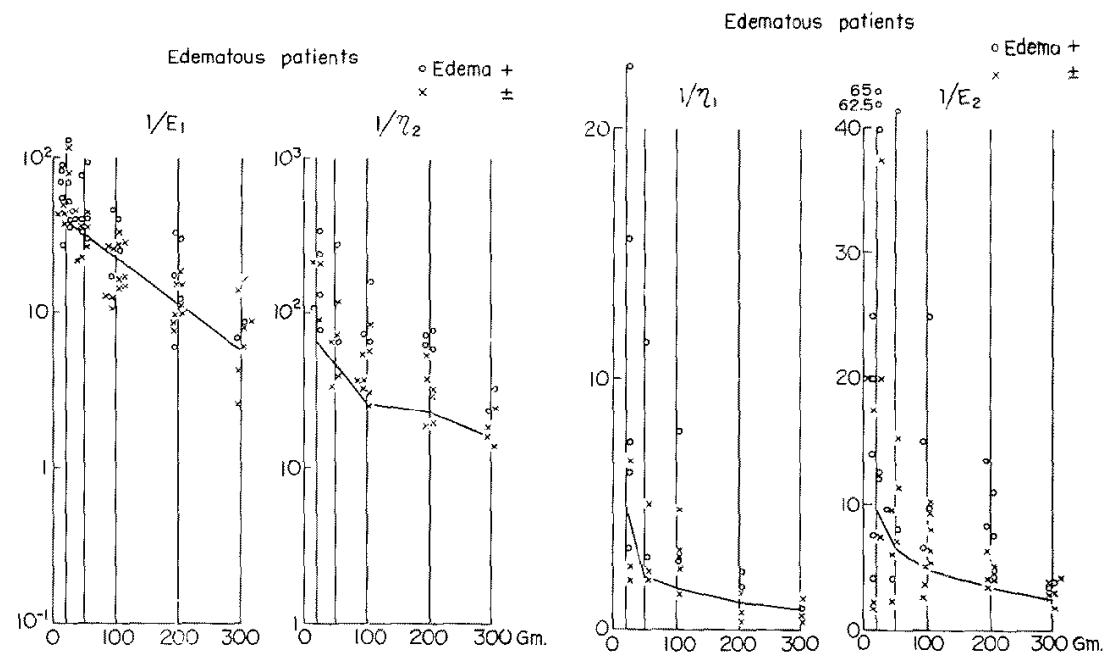

Fig. 9a. Distribution of $1 / E_{1}$ and $1 / y_{2}$ in human female.

b. Distribution of $1 / \eta_{1}$ and $1 / E_{2}$ in human female.

normal skin tissue, and hence $\eta_{1}$ and $\eta_{2}$ are largely different from control values. But it is somewhat surprising to find that $E_{\Omega}$ also slips out from normal range. According to our model (Fig. 4) this means that limiting force for deformation and spring for reformation against $\eta_{2}$ is weakened. It may be that tissue meshwork holding normal shape is deluged in edematous patients. $\mathrm{E}_{1}$ is unchanged by the presence of the edema.

In 2 heavily edematous patients, we had chance to measure moduli again after complete regression of edema (Fig. 10a and b). One 56 year 

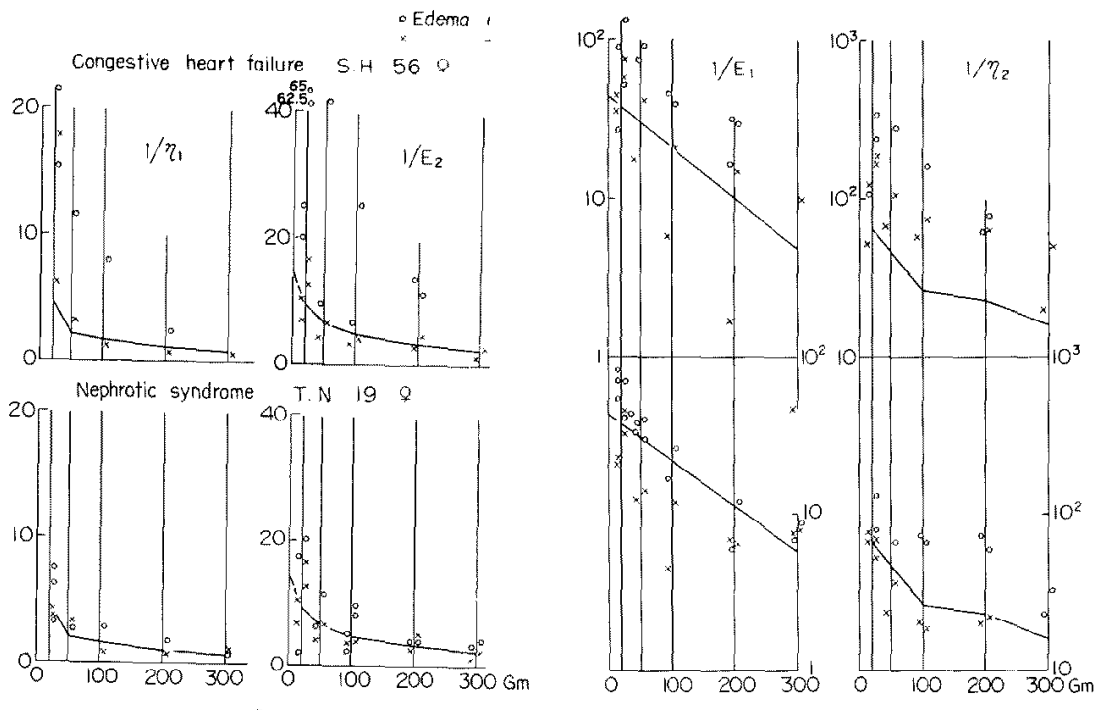

Fig. 10. Comparison of viscoelastic moduli during edema and after its disappearance.

old woman had congestive heart failure due to mitral stenosis and pulmonary fibrosis, and the other 19-year-old girl had nephrotic syndrome. By comparison $\eta_{1}$ was greatly dislocated at first in congestive heart failure, while nearly normal in nephrotic syndrome. After edema has dissipatcd in the former case the modulus was normalized. $\mathrm{E}_{2}$ showed the same tendency. $\mathrm{E}_{1}$ scemed unchanged in both patients. There was, however, some discrepancy in $\eta_{2}$, too, for congestive failure showed no improvement but nephrotic patient ameliorated modulus to certain extent. It may be said that in congestive heart failure tissue shape-holding power is restored largely by $\mathrm{E}_{2}$ and velocity factor $\eta_{2}$ shows little improvement, and that in nephrotic syndrome the reverse is seen.

\section{Discussion}

The skin tissue over the tibial margin may be the simplest of the body tissue for viscoelastic measurement, but histologically the skin is also comprised of many components. Each component may have its own characteristics and we can measure only the gross summation of these characteristics. Schade was well-informed at that age of many viscoelastic phenomena and their significance. ${ }^{1)}$ But he used only circulating gauge and could read the dial at intervals. His elasticity-loss seems to us that he measured $\eta_{1}$ in reality. We think this modulus represents capillary filtrability. The reason for this is mentioned later. He also used relaxation phenomenon for discrimination of the edema. It corresponds mainly to 
Maxwell element or upper part of our model, and is due to $\eta_{1}$ as its major participant.

Watanabe read the gauge dial at shorter intervals, and found that fluidity of the skin tissue is more important than Schade thought. ${ }^{2}$ He measured the proportion of displacement due to $\mathrm{E}_{1}$ caused by test weight in the total displacement after 2 minutes. According to our opinion his index showed the importance of $\gamma_{1}$ and $E_{2}$ in alterations found in edematous skin. The implication of his index is broken into 2 moduli in our analysis, and the latter is interpreted as tissue meshwork serving to hold the shape of the tissue.

Rheology has already its application in many fields of biology and medicine. It has many formulations in its mathematical style. For example, Reiner preferred his tensor expression, ${ }^{4}$ and Bland developed 3-dimensional model.5) They have their causes by applicability to real bodies. We feel for objects such as skin tissue in which dislocation of water is under question, one-dimensional simple treatment is sufficient. But even in simple one-dimensional formulation, real responses of living tissue may be complex enough. As described in methods there was often need for considering a more generalized viscoelastic model. We will show some specimens for such responses, one from edematous patient and the other from non-edematous patient (Fig. 1la and b). Calculated approximately from rod cross-sectional area $7 \mathrm{~mm} .^{2}$ and tissue thickness $7 \mathrm{~mm}$, we obtain some $4-8 \mathrm{ml}$. per $100 \mathrm{Gm}$. skin tissue is dislocated by quicker component. It is too large for its blood content. So at this moment we can not identify this component in morphological terminology.

Now we have to consider the substantial meanings of our moduli. $E_{1}$ is elastic componet of the skin, and we think blood in greater vessels may be included in this component because of its easy mobility. $\eta_{1}$ is a factor which comes into effect only gradedly by application of external force. Removing of the test weight may remain the deformation. Only if negative pressure is applied, it returns to original starting point. We speculate it to be index for the capillary filtration. From calculations as above, it represents $0.007 \mathrm{ml}$. per $100 \mathrm{Gm}$. tissue per $1 \mathrm{~mm} . \mathrm{Hg}$ external pressure in 1 minute. It agrees well with vaiues of Landis in human $(0.006 \mathrm{ml} .)^{6)}$ or of Pappenheimer in experimental animals. ${ }^{7)} \mathrm{E}_{1}$ and $\eta_{1}$ comprise Maxwell element of the skin model.

$\mathrm{E}_{\Omega}$ and $\eta_{2}$ are moduli comprising Voigt (or Kelvin) element of the model. It acts as shape-holding power of the skin. When external force is applied it reacts retardedly to final deformation range, and when test weight is removed it again reacts slowly towards reformation. It shows the compactness of the skin tissue. $E_{1}$ and $E_{2}$ are both elastic com- 

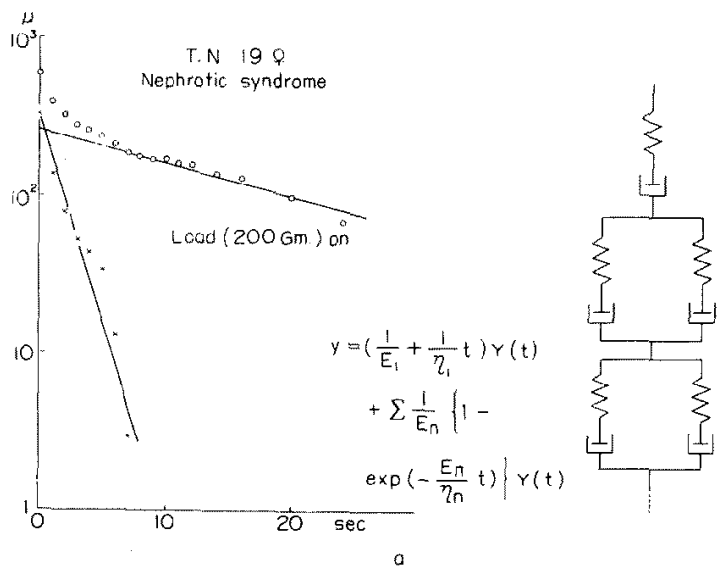

Fig. Ila. Gencralized viscoelastic model for skin.
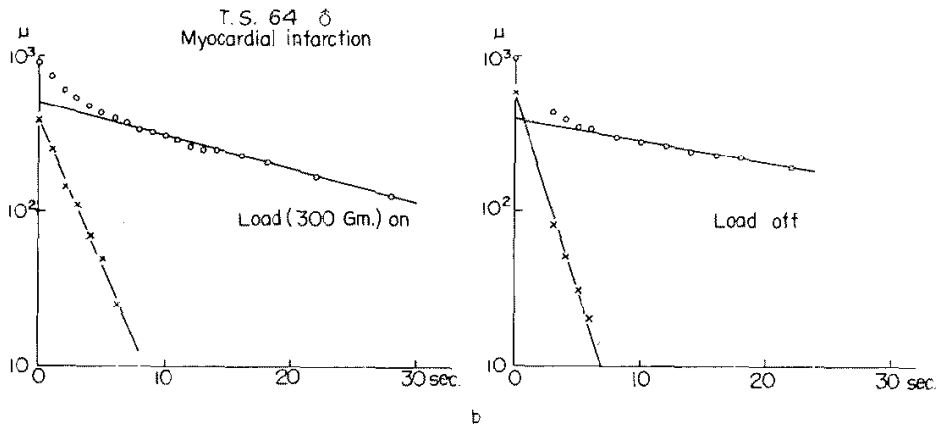

Fig. 11b. Gencralized viscoclastic model for skin.

ponents. It may not be different substance in the skin. According to such a consideration, it may be said that only about one third of the elastic component of the skin participates in shape-holding activity. In our unpublished data in a patient with scleroderma, $\mathrm{E}_{1}$ was the sole modulus under derangement leaving other moduli unaffected. So there remains problem whether $\mathrm{E}_{1}$ and $\mathrm{E}_{2}$ represent heterogeneous components of the skin. As to $r_{2}$ we consider water molecule mobility in the tissuc space.

In edematous patients, generally speaking, $\eta_{1}$ is decreased, while $E_{1}$ is in normal range. According to our interpretation $\eta_{1}$ signifies capillary filtrability. So this decrease means that there is increased tendency to capillary filtration in edematous patients. Smirk also demonstrated increased capillary filtrability in his patients by oncometry. ${ }^{8}$ But his method is very time-consuming, and if our method is approved this is much convenient. Theoretically, there remains one problem for applicability of our method in patients. That is when some $\mathrm{E}$ of Voigt elements (in generalized model) becomes so small, some $\eta$ of this element might 
merge into $\gamma_{1 /}$ of our measurement. Except this apprehension our method is practicable. $E_{2}$ and $\gamma_{2}$ show decreased tissue compactness or easy susceptibility for deformation in edematous patients. After dissipation of the edema, tissue resumes its compactness and quickly regains its original shape after the test. As shown in results (Fig. 10a and b) there may exist some difference in alteration in moduli according to the etiology. It should have to be accounted for by corresponding tissue pathology. But at the present time we withhold for some while for further comprehension until more data will be accumulated.

\section{Summary}

A continuously recording apparatus is devised to measure the deformation of skin surface on tibial margin against test weight. The deformation observed by this apparatus is best approximated by 4 element viscoelastic model in rheology. One of the moduli, $\eta_{1}$, is thought to represent capillary filtrability. It is calculated that capillary filtration in non-edematous patients is about $0.007 \mathrm{ml} .100 \mathrm{Gm}$. tissue $/ \mathrm{mm} . \mathrm{Hg} / \mathrm{min}$.

In edematous patients there are seen profound alterations in these moduli, and increased capillary filtrability and easy susceptibility to deformation are expressed numerically. After dissipation of edema these alterations usually return to normal range.

We wish our method to be called tissue rheometry.

\section{References}

1. Schade, H.: Münch. Med. Wschr., 73: 2241, 1926.

2. Watanabe, N.: J. Jap. Soc. Int. Med. 41: 714, 1953 (In Japanese).

3. Yoshitoshi, Y. and Nagasaka, M. : Jap. J. Nephrol. (In Japanese) (In press).

4. Reiner, M. : Lectures on Theoretical Rheology, Amsterdam, 1960.

5. Bland, D. R.: The Theory of Linear Viscoelasticity, 1960.

6. Krogh, A., Landis, E. M., and Turner, A. H.: J. Clin. Invest. 11: 63, 1932.

7. Renkin, E. M. and Pappenheimer, J.R.: Erg. Physiol. 49: 59, 1957.

8. Smirk, F. H.: Clin. Sc. 2: $317,1936$. 